\title{
Commentary: Three-dimensional P3 tethering angle at the heart of future surgical decision making in ischemic mitral regurgitation
}

\author{
Wobbe Bouma, MD, PhD, ${ }^{\mathrm{a}, \mathrm{b}}$ and Robert C. Gorman, $\mathrm{MD}^{\mathrm{a}}$
}

In complex mitral valve disease, such as ischemic mitral regurgitation (IMR), different mechanisms go on to produce IMR to different extents and the relative contributions of these mechanisms may change over time, partially due to the delicate interrelated geometry of the mitral valve. ${ }^{1}$ In a rather sophisticated study employing 3-dimensional echocardiography (3DE), Sun and colleagues ${ }^{2}$ show that the 2 main pathophysiologic mechanisms of IMR; that is, annular dilatation and flattening (Carpentier type I) and posterior leaflet (segment P3) tethering due to left ventricle dilatation (Carpentier type IIIb), both independently predict moderate IMR improvement 1 year after coronary artery bypass grafting (CABG). Receiver-operating characteristic analysis showed that a nonplanar angle $<158.1^{\circ}$ and a P3 tethering angle $(\mathrm{P} 3 \mathrm{TA})<28.8^{\circ}$ reliably predict IMR improvement after $\mathrm{CABG} .{ }^{2}$ This is an important finding because the presence of IMR significantly worsens prognosis ${ }^{3}$ yet a beneficial effect on prognosis for mitral valve surgery in addition to CABG for moderate IMR has never been shown. ${ }^{4}$ Thus, when reliable predictors are established that advocate for declining to intervene on moderately regurgitant ischemic mitral valves of certain subgroups it is of huge clinical and financial consequence.

Sun and colleagues ${ }^{2}$ show, in an off-pump CABG cohort, that remodeling effects after coronary revascularization may attenuate tethering and IMR in select patients. Although off-pump CABG can be advantageous for select patients, it seems unlikely that the coronary revascularization technique (ie, on pump vs off pump) would influence IMR improvement. However, it is a limitation of the study and more work is needed in larger, more heterogeneous cohorts to determine this with more certainty.

The 3DE imaging and modeling in the study by Sun and colleagues $^{2}$ are based on methods that were introduced by

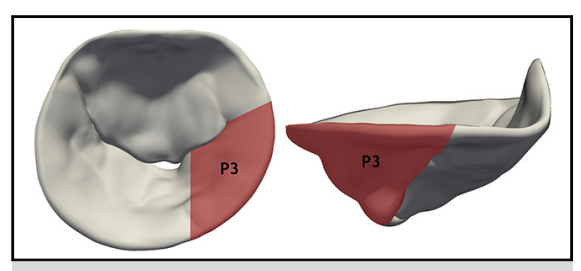

3DE P3TA may be at the heart of future surgical decision making in IMR.

\section{Central Message}

Preoperative 3DE P3TA may be at the heart of more refined surgical decision making in ischemic mitral regurgitation.

See Article page 1795 .

our group. ${ }^{5}$ Additional studies from our group with similar modeling algorithms have shown that preoperative $3 \mathrm{DE}$ P3TA is a strong (ie, independent) predictor of IMR recurrence after undersized ring annuloplasty for severe $\mathrm{IMR}^{6}$ and that $3 \mathrm{DE}$ is superior over 2-dimensional echocardiography because it is not dependent on viewing plane selection. ${ }^{7}$ Those studies and the study by Sun and colleagues $^{2}$ are, however, limited by the fact that IMR severity was measured semiquantitatively with jet area/left atrium area. Future IMR studies should include quantitative IMR severity assessment with effective regurgitant orifice area and regurgitant volume.

On a day-to-day basis, cardiac surgeons are faced with 2 important questions regarding IMR: Should we operate on this (moderately) regurgitant mitral valve and, Should we repair or replace this (severely) regurgitant mitral valve? As shown by Sun and colleagues ${ }^{2}$ and by our group,,${ }^{6,7}$ 3DE P3TA may be the key to answering these questions. At this point, both questions are difficult to answer for an individual patient based on current guidelines. In the 2017 American Heart Association/American College of Cardiology focused update of the 2014 guidelines, mitral valve repair is no longer recommended for patients with chronic moderate IMR undergoing CABG ("the usefulness is uncertain"; class IIb recommendation [weak], level of evidence B [randomized]) and it is reasonable to choose chordal-sparing mitral valve replacement over repair in patients with chronic severe IMR (class IIa recommendation [moderate], level of evidence B [randomized]). ${ }^{8}$ The current 
guidelines provide generalized statements on patients with IMR and do not account for patient subgroups with milder or more extreme forms of tethering. In an effort to do so, the American Association of Thoracic Surgery updated its consensus guidelines on IMR and now identifies subgroups based on (amongst other factors) tethering. ${ }^{9}$ At this point, we are in need of studies such as that performed by Sun and colleagues $^{2}$ to pave the way for more refined evidence-based guidelines on surgical treatment of IMR. Future predictive models should not only include geometric and strain-related factors but also clinical patient-related factors (including age, comorbidity, and left ventricular function) and procedure-related factors (including the risk of surgery and the risk of repair failure with prolonged cardiopulmonary bypass and aortic crossclamping) to decide between surgery or no surgery or between a sophisticated custom-made repair or chordal-sparing mitral valve replacement. Although there is still a long road ahead before we can refine the guidelines, it is becoming more and more clear that 3DE P3TA may be at the heart of more refined future surgical decision making in IMR.

\section{References}

1. Bouma W, van der Horst ICC, Wijdh-den Hamer IJ, Erasmus ME, Zijlstra F, Mariani MA, et al. Chronic ischaemic mitral regurgitation. Current treatment results and new mechanism-based surgical approaches. Eur J Cardiothorac Surg. 2010;37:170-85

2. Sun X, Jiang Y, Huang G, Huang J, Shi M, Pang L, et al. Three-dimensional mitral valve structure in predicting moderate ischemic mitral regurgitation improvement after coronary artery bypass grafting. J Thorac Cardiovasc Surg. 2019; 157:1795-803.e2.

3. Grigioni F, Enriquez-Sarano M, Zehr KJ, Bailey KR, Tajik AJ. Ischemic mitral regurgitation: long-term outcome and prognostic implications with quantitative Doppler assessment. Circulation. 2001;103:1759-64.

4. Michler RE, Smith PK, Parides MK, Ailawadi G, Thourani V, Moskowitz AJ, et al Two-year outcome of surgical treatment of moderate ischemic mitral regurgitation. $N$ Engl J Med. 2016;374:1932-41.

5. Ryan LP, Jackson BM, Enomoto Y, Parish L, Plappert TJ, St JohnSutton MG, et al. Description of regional mitral annular nonplanarity in healthy human subjects: a novel methodology. J Thorac Cardiovasc Surg. 2007; $134: 644-8$.

6. Bouma W, Lai EK, Levack MM, Shang EK, Pouch AM, Eperjesi TJ, et al Preoperative three-dimensional valve analysis predicts recurrent ischemic mitral regurgitation after mitral annuloplasty. Ann Thorac Surg. 2016;101: 567-75.

7. Wijdh-den Hamer IJ, Bouma W, Lai EK, Levack MM, Shang EK, Pouch AM, et al The value of preoperative 3-dimensional over 2-dimensional valve analysis in predicting recurrent ischemic mitral regurgitation after mitral annuloplasty. $J$ Thorac Cardiovasc Surg. 2016;152:847-59.

8. Nishimura RA, Otto CM, Bonow RO, Carabello BA, Erwin JP III, Fleisher LA, et al. 2017 AHA/ACC focused update of the 2014 AHA/ACC guideline for the management of patients with valvular heart disease: a report of the American College of Cardiology/American Heart Association task force on clinical practice guidelines. Circulation. 2017;135:e1159-95.

9. AATS Ischemic Mitral Regurgitation Consensus Guidelines Writing Committee Kron IL, LaPar DJ, Acker MA, Adams DH, Ailawadi G, Bolling SF, et al. 2016 update to The American Association for Thoracic Surgery (AATS) consensus guidelines: ischemic mitral valve regurgitation. J Thorac Cardiovasc Surg. 2017;153:e97-114. 